\title{
Flash chromatography system: a practical tool for demonstrating the influence of column characteristics on chromatographic resolution.
}

Amaury Kasprowiak ${ }^{a}$, Francine Cazier-Dennina,b, Pierre-Edouard Danjou,a,b,*

a Département de Chimie, Université du Littoral Côte d'Opale, 220 avenue de l'université, Dunkerque, France

b Unité de Chimie Environnementale et Interactions sur le Vivant, EA 4492, Université du Littoral Côte d'Opale, 145 Avenue Maurice Schumann, MREI 1, Dunkerque, France

*danjou@univ-littoral.fr

\section{ABSTRACT}

A flash purification system using prepacked cartridges containing different silica weight and particle sizes was employed to demonstrate the influence of column characteristics on chromatographic resolution. This chromatographic experiment was a helpful introduction to chromatographic courses where the influence of particle sizes was addressed only theoretically and it allows students to experience the concept in an active learning style experiment. After running a predefined set of experiments to understand concepts, students were asked to design their own experiment to achieve the best chromatographic resolution. Moreover, the difference of mass versus concentration detectors was highlighted using Ultraviolet (UV) and Evaporative Light Scattering Detectors (ELSD). Questionnaires were also used to define the students' initial knowledge and to assess on what they learned following this lab. 


\section{GRAPHICAL ABSTRACT}

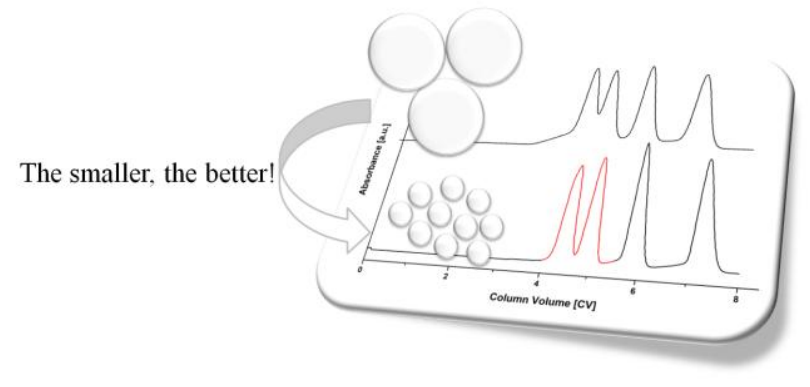

\section{KEYWORDS}

Chromatography, Analytical Chemistry, Laboratory Instruction, Hands-On Learning/Manipulatives, Upper-Division Undergraduate, Laboratory Equipment / Apparatus

The concept of flash chromatography was first introduced in 1978 with the promise of separating several grams of compound in a very short time ${ }^{1}$ (15 minutes instead of 2-3 hours). While this technique is commonly used by organic chemists, it has not undergone any major evolution over several decades and remains the method of choice for the purification of organic compounds. Some minor modifications were proposed to facilitate access to this technique in teaching laboratories $2,3,4$. However, the emergence of automated flash chromatography systems like Reveleris (Buchi) ${ }^{5}$, Isolera (Biotage) ${ }^{6}$ or Puriflash (Interchim) ${ }^{7}$, has deeply changed the ways compounds are purified and open new teaching opportunities in the organic synthesis as well as in analytical chemistry. It is obvious that such devices will find their usefulness in the organic chemistry laboratory in complement/replacement of traditional column chromatography ${ }^{8}$ since it became increasingly popular in companies as well as in academic research labs. Moreover, the use of prepacked silica cartridges prevents the handling of hazardous silica gel $^{9}$ by the students, leading to a safer experiment.

Nevertheless, it could also be interesting to introduce flash chromatography systems in a chromatography module as it gives access to a large variety of prepacked cartridges of different sorbent weights, particles diameters, and phase nature (normal, reverse, grafted, chiral phases etc.). Sorbent weights could easily be related to HPLC column length and/or diameter since in both cases it presents an increase in active site number. It also gives the students notions regarding HPLC column choice. This variety of cartridges allows the study of other parameters than those commonly studied in typical liquid chromatography labs that are mainly focused on the influence of mobile phase composition on chromatographic separation. Moreover, some flash chromatography systems possess two different detectors, namely Ultraviolet (UV) and Evaporative Light Scattering (ELSD) Detectors, allowing students to compare the influence of the detection mode on chromatograms acquired during the same run. 
The influence of some parameters like silica weight (i.e. column loading), particle sizes ${ }^{10}$, type of detectors $^{11}$ is often taught theoretically but students are rarely offered the possibility to demonstrate their influences during a lab session. This lack of practical experience is due to the fact that it is not common to own traditional HPLC columns in different lengths and silica particle sizes. Moreover, most of the time, HPLC only have one detector ranging from the most common UV-visible detector ${ }^{7,12,13}$ to the most advanced (and expensive) tandem mass spectrometer. ${ }^{14,15}$

To address this lack of practical demonstration, we have designed a laboratory experiment (Figure 1) in which students are invited to experience different chromatographic parameters in an active learning style experiment thanks to an automated flash chromatography system. To the best of our knowledge, it is the first time that a flash chromatography device is used for teaching purposes.

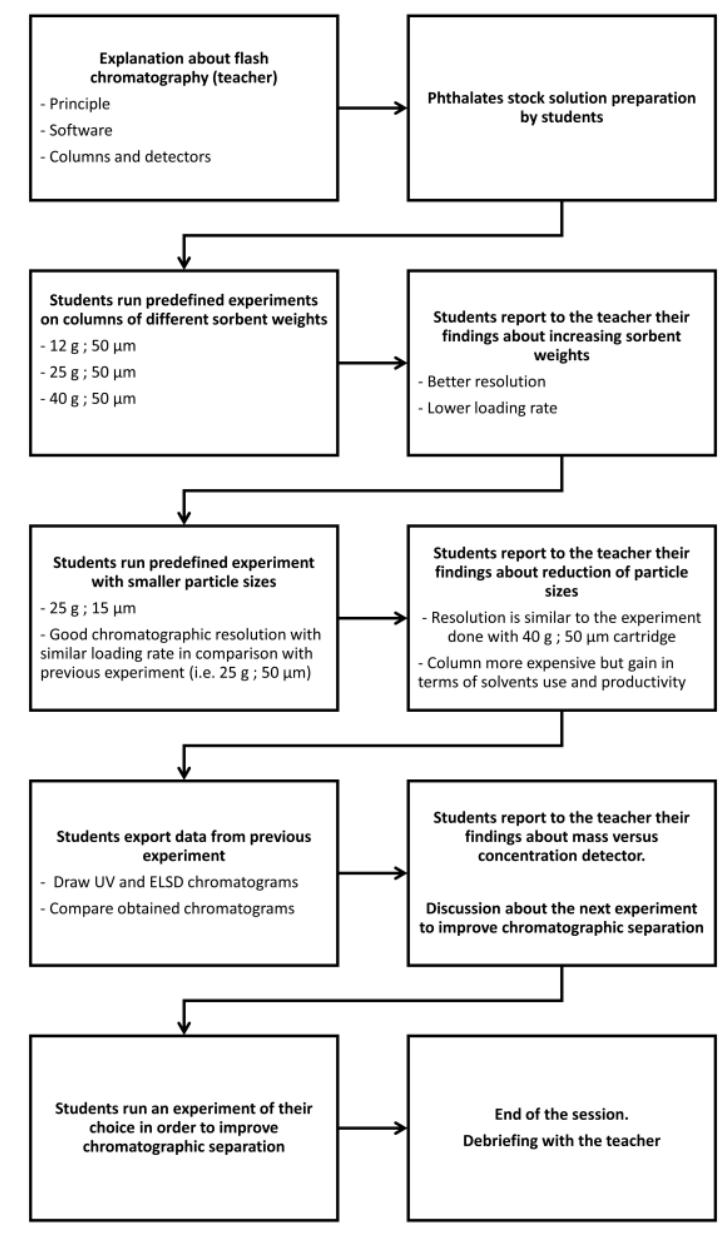

Figure 1 : Flow diagram of the lab session 


\section{LEARNING OUTCOMES}

At the end of the lab session, students are able to:

(1) Explain the operation of a flash chromatography system

(2) Separate a mixture of compounds using flash chromatography system

(3) Explain the influence of sorbent weight as well as silica particle sizes on chromatographic resolution

(4) Conduct a scale change using Column Volume (CV)

(5) Predict elution order on normal phase chromatography and compare with reverse phase HPLC

(6) Explain the difference between mass and concentration detector ${ }^{16}$

\section{EXPERIMENTAL PROCEDURE}

Apparatus description

The flash chromatography device used in this study is a classical commercial apparatus incorporating a quaternary gradient pump system, diode array detector $(200-600 \mathrm{~nm})$ allowing to monitor up to 4 wavelengths simultaneously and a full UV spectrum as well as an Evaporative Light Scattering Detector (ELSD) for universal detection.

Column choice

Gradient optimization was performed on prepacked silica cartridges loaded with $25 \mathrm{~g}$ of $50 \mu \mathrm{m}$ silica particles. Using the method of Column Volumes (CV), the gradient was transferred to cartridges with different dimensions and sorbent loadings (i.e., 12, 25 and 40 g). The same gradient was applied on the $15 \mu \mathrm{m}$ silica particles at a $25 \mathrm{~g}$ loading. Cartridges were reused several times (more than 5 times) during the same day without efficiency loss. As stated by the supplier and experienced by us, shelf-life of a column under hexane does not exceed one day.

Stock phthalate solution

Using a volumetric flask and ethyl acetate (analytical grade), students prepared $10 \mathrm{~mL}$ of stock solution containing $2 \mathrm{~g}$ of each dialkyl phthalates: dimethylphthalate (DMP, >99\%), diethylphthalate (DEP, 99.5\%), dipropylphthalate (DPP, 98\%), dibuthylphthalate (DBP, 99\%) This solution was used for each experiment. 
To avoid bias in comparison between column, students were asked to apply the following gradient

(Table 1) expressed in Column Volume (CV) instead of time. During optimization, it was found that an adequate but not perfect resolution could be achieved in about $8 \mathrm{CV}$.

Table 1. Elution conditions

\begin{tabular}{ccc} 
CV & n-hexane & Ethyl acetate \\
0 & 100 & 0 \\
5 & 80 & 20 \\
8 & 80 & 20 \\
\hline
\end{tabular}

\section{HAZARDS}

Since the experiment requires manipulation of organic solvents and compounds as well as a potentially high-pressure device, students must wear appropriate protective equipment i.e. lab coat, goggles, and gloves. Ethyl acetate and n-hexane are highly inflammable, irritant liquids, and should be manipulated with care in a well-ventilated fume hood. n-hexane is neurotoxic, suspected of damaging fertility, and toxic to aquatic life. Solvent should be disposed properly. Phthalates are potentially toxic for human reproduction ${ }^{17}$ and should be manipulated with care.

\section{RESULTS}

The first set of experiments was performed using cartridges containing $50 \mu \mathrm{m}$ spherical silica in order to demonstrate the influence of column loading on the resolution (Table 2, entry 1-3). Gradient, injection volume and concentration of phthalates in solution remained constant for all experiments. The optimal flow rate for $40 \mathrm{~g}$ cartridge was chosen according to manufacturer data (entry 3 ) instead of the same $15 \mathrm{~mL} / \mathrm{min}$ flow for all cartridges since no differences were evidenced between 26 and 15 $\mathrm{mL} / \mathrm{min}$ (see supplementary data). All chromatograms were expressed in Column Volume (CV) as it allows to compare data recorded on cartridges of different sorbent weights. To be precise, retention times are dependent on flow rate and sorbent weight while CV are not affected by such parameters and stay relatively stable. Another advantage of the use of $\mathrm{CV}$ is that it makes it easy to conduct a change of scale, either up or down, in flash chromatography. From Figure 2, it can be seen that $12 \mathrm{~g}$ of $50 \mu \mathrm{m}$ silica led to a poor separation of all phthalates, particularly DBP and DPP which are clearly coeluted. Increasing silica weight to $25 \mathrm{~g}$ allows us to discern the four different analytes with a resolution of only 0.76 for the first two peaks (DBP and BPP). This gain in resolution can be related to the 
increase in number of theorical plate which accompany increase in column length. As expected, a far better resolution (1.08) was obtained using a $40 \mathrm{~g}$ silica cartridge (entry 3) which results in a decrease in column loading. Resulting chromatograms were presented on Figure 2.

Table 2. Experiment Overview

\begin{tabular}{|c|c|c|c|c|c|c|c|c|c|}
\hline \multirow[t]{2}{*}{ Entry } & \multicolumn{3}{|c|}{ Cartridge Conditions } & \multirow{2}{*}{$\begin{array}{l}\mathrm{CV}_{0} \\
\mathrm{~mL} a\end{array}$} & \multirow{2}{*}{$\begin{array}{l}\text { Optimal } \\
\text { Flow, } \\
\text { mL/min }\end{array}$} & \multirow{2}{*}{$\begin{array}{c}\text { Column } \\
\text { Loading, } \\
\%^{b}\end{array}$} & \multicolumn{3}{|c|}{ Resolutions $^{c}$} \\
\hline & $\begin{array}{c}\text { Sorbent } \\
\text { Weight, g }\end{array}$ & $\begin{array}{c}\text { Particle Size } \\
\mu \mathrm{m}\end{array}$ & $\begin{array}{l}\text { Internal Diameter } \\
\times \text { Length, } \mathrm{mm}\end{array}$ & & & & DBP/DPP & DPP/DEP & $\mathrm{DEP} / \mathrm{MP}$ \\
\hline 1 & 12 & 50 & $21 \times 78$ & 20.5 & 15 & 6.6 & $\mathrm{ND}^{d}$ & $\mathrm{ND}^{d}$ & 1.16 \\
\hline 2 & 25 & 50 & $21 \times 127$ & 32.5 & 15 & 3.2 & 0.76 & 1.41 & 1.92 \\
\hline 3 & 40 & 50 & $27 \times 127$ & 52.0 & 26 & 2.0 & 1.08 & 1.85 & 2.35 \\
\hline 4 & 25 & 15 & $21 \times 127$ & 32.5 & 15 & 3.2 & 1.13 & 2.68 & 2.95 \\
\hline
\end{tabular}

aColumn Volume according to manufacturer data. $b$ Weight of analyte/weight of silica. ${ }^{c}$ Resolutions were determined graphically. ${ }^{d}$ Not determined due to DBP and DPP coelution.

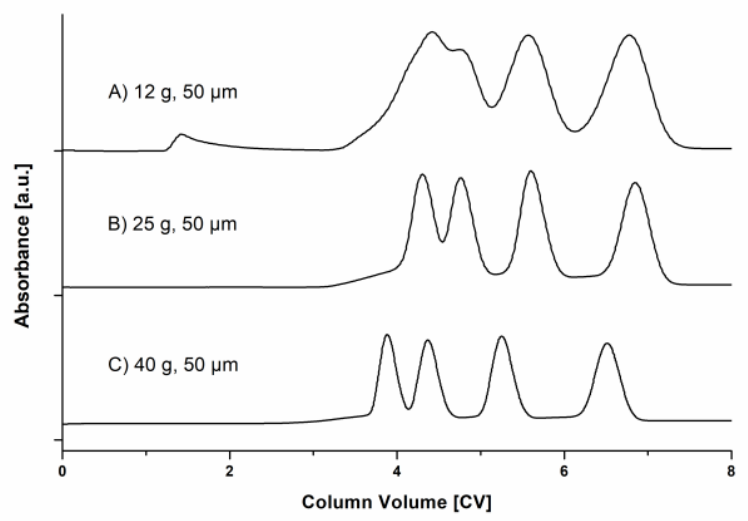

Figure 2 : Separation of phthalates using different silica sorbent weights with 50 $\mu \mathrm{m}$ silica particles. A) 12 g, B) 25 g, and C) 40 g. Conditions: Cartridges and flow rates shown in Table 2, ambient temperature, 1 mL injection, $254 \mathrm{~nm}$ detection. Elution order: DBP, DPP, DET, DMP.

Afterwards, an experiment was run using $25 \mathrm{~g}$ of $15 \mu \mathrm{m}$ spherical silica in order to visualize the effect of particle sizes diminution on the chromatographic resolution. Erreur! Source du renvoi introuvable., entry 2 and 4 experiments were presented Figure 3. Students can then easily notice a better resolution (1.13) between DBP and DPP on this cartridge (25 g, 15 $\mu \mathrm{m}$ silica), similar to the one obtained with $40 \mathrm{~g}$ cartridge $(50 \mu \mathrm{m})$. At this point, the students were able to understand that if the CV remains fairly constant for all the compounds, the volume of solvent used is $40 \%$ lower on the 
cartridge containing $25 \mathrm{~g}$ of silica $(260 \mathrm{~mL}$ against $416 \mathrm{~mL}$ ) leading to a more practical and economical separation with smaller silica particles.

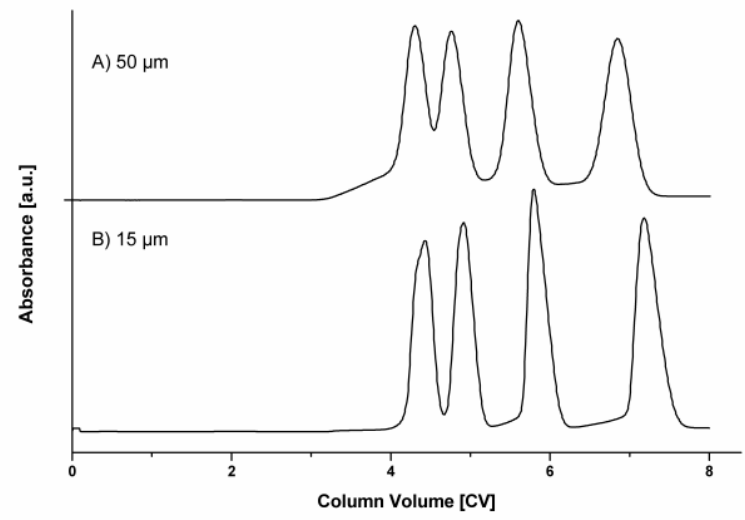

Figure 3 : Separation of phthalates using different silica particles sizes at constant sorbent weight (25g). A) $50 \mu \mathrm{m}$, B) $15 \mu \mathrm{m}$. Conditions: $21 \times 130 \mathrm{~mm}$ column, $15 \mathrm{~mL} / \mathrm{min}$ flow, ambient temperature, $1 \mathrm{~mL}$ injection, $254 \mathrm{~nm}$ detection. Elution order: DBP, DPP, DET, DMP.

A comparison of responses between the UV-detector $(254 \mathrm{~nm})$ and ELSD recorded during run 4 from table 2 was presented in Figure 4. It can be observed that the UV-detector provides a similar intensity of response for the four phthalates analytes while a great disparity was observed with ELSD leading to a poor signal for the smallest compound (DMP). This can be explained by the fact that in UV detection the signal is proportional to the analyte's concentration according to the Beer-Lambert's law while ELSD is sensitive to molecules sizes after solvents' evaporation, leading to, in general, intense signal for larger compounds. ${ }^{18}$

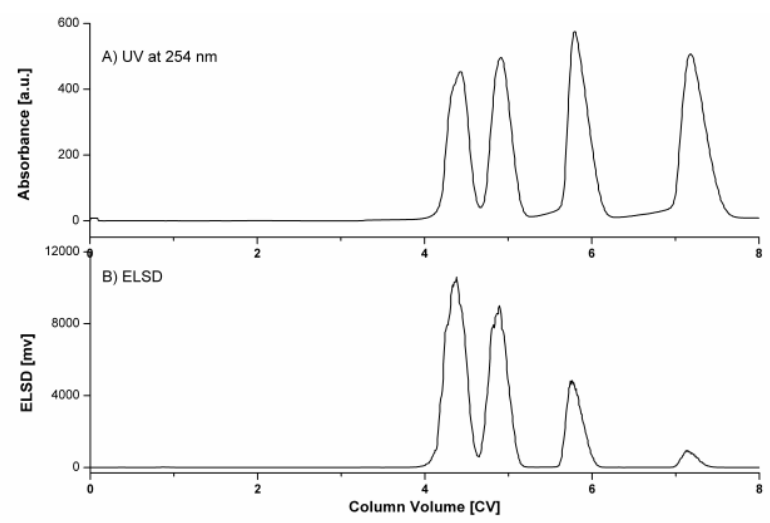

Figure 4 : Response of detectors during the separation of phthalates. A) UV at 254 nm, B) ELSD. Conditions: $21 \times 130 \mathrm{~mm}$ column, $25 \mathrm{~g}$ silica, $15 \mu \mathrm{m}$ particle sizes, $15 \mathrm{~mL} / \mathrm{min}$ flow, ambient temperature, $1 \mathrm{~mL}$ injection, Elution order: DBP, DPP, DET, DMP. 


\section{DISCUSSION}

This 3 hours laboratory experiment was performed in autumn 2018 and autumn 2019 with master's degree students in analytical chemistry in addition to their traditional chromatography laboratory classes dealing with paraben identification by reverse-phase HPLC-UV and PAH quantification in polluted soil by GC-FID. The education and curricula of the students are diverse since some of them only know chromatography through TLC, others spend about 10 to $20 \mathrm{~h}$ in GC-FID, HPLC-UV and ion chromatography labs while a few of them spend a 3-month training period in the industry on a chromatograph. Those differences in student's knowledge explain why the chromatography program in our formation starts from scratch and why we choose to perform this module with graduate students and not undergraduate students. We believe that undergraduate courses dealing with chromatography theory could also benefit from this experiment.

The pedagogical staff decided to offer this lab session to students at the beginning of the chromatography courses in order to promote discovery learning of some chromatographic parameters that will be taught later in the semester. It enriched the students' curricula by incorporating notions in normal phase chromatography, flash chromatography and ELSD detector. Students were asked to answer the same questionnaire (see supplementary data) before and after the lab session to evaluate their initial knowledge and learning. It is important to mention that the first questionnaire was completed while students didn't yet receive instructions for the lab session in order to truly evaluate their initial knowledge. A compilation of their answers was presented in table 3.

\section{Table 3. Distribution of Students' Responses to the Pre-Lab Questionnaire}

\begin{tabular}{|c|c|c|c|c|c|}
\hline \multirow{2}{*}{ Item } & \multirow[t]{2}{*}{ Questions for Response } & \multicolumn{3}{|c|}{ Student Responses, $N=20$} & \multirow[t]{2}{*}{ Remarks } \\
\hline & & Yes, \% & No, $\%$ & No Answer, \% & \\
\hline 1 & Had you ever used a liquid chromatograph? & 60 & 40 & 0 & \\
\hline 2 & Had you ever heard of ELSD? & 5 & 95 & 0 & None could explain \\
\hline 3 & $\begin{array}{l}\text { Does the size of the silica particles in a column } \\
\text { affect the chromatogram? }\end{array}$ & 50 & 0 & 50 & Only $10 \%$ could explain \\
\hline 4 & $\begin{array}{l}\text { Before the lab had you ever heard of flash } \\
\text { chromatography? }\end{array}$ & 40 & 60 & 0 & Only $30 \%$ could explain \\
\hline 5 & What type (s) of detector (s) did you use? & $45^{a}$ & $5^{b}$ & 50 & \\
\hline 6 & What is the magnitude measured on the abscissa? & $70^{c}$ & $5^{d}$ & 20 & \\
\hline
\end{tabular}


Before the lab session, $60 \%$ of students never performed HPLC (Table 3 entry 1) and only $45 \%$ of students were able to mention UV as HPLC detector. The student who proposed the conductimetry was certainly confused with ion chromatography. The students probably guessed from the question that particle size affects the chromatogram (entry 3 ) but only $10 \%$ can explain how. No students were aware of the difference between mass and concentration detectors nor the existence or principle of the ELSD (entry 2). However, most of the students knew that the abscissa value on a chromatogram was the time (entry 6).

At the end of the lab session, all the students were able to mention in the questionnaire i) the type of detectors they employed (UV and ELSD) and if it was a mass or concentration detector ii) that particle sizes influence the chromatogram and how iii) that the abscissa value on a chromatogram could be expressed in time or Column Volume. Those results allow us to conclude that all the learning outcomes previously described were reached. A parallel was done during lecture between students' skill acquired during this lab session and the advantages of particle sizes diminution in UPLC as well as the difference between mass and concentration detectors. This parallel allows students to better understand those parameters that may seem abstract at first glance.

During the lab session students were asked to find a way to prove the elution order using common techniques. Among the responses, students proposed the following solutions:

i) Inject standards compounds one by one

ii) Add more weight of a particular phthalate in the existing solution (standard addition)

iii) Collect pure fraction from the column and evaporate the solvent under reduced pressure to obtained pure compound that can be analyzed by infrared spectroscopy, NMR spectroscopy, refractive index or mass spectrometry.

Students were also asked how they can improve the chromatographic separation of the same mixture and to experiment with their proposal. The following solutions were proposed by students and tested during the lab session (see supplementary data):

i) Increase sorbent weight with $15 \mu \mathrm{m}$ silica particles (i.e. $40 \mathrm{~g}$ silica cartridge with $15 \mu \mathrm{m}$ particle sizes) 
ii) Modifying the gradient (i.e. Ethyl acetate gradient in $8 \mathrm{CV}$ instead of $5 \mathrm{CV}$ )

iii) Inject less solution (i.e. $0.5 \mathrm{~mL}$ instead of $1 \mathrm{~mL}$ )

Among those solutions, the best resolutions of 1.50 and 1.73 between DBP and DPP were obtained by injecting $0.5 \mathrm{~mL}$ of the phthalates solution on cartridge containing $15 \mu \mathrm{m}$ silica particle sizes with a silica weight of $25 \mathrm{~g}$ and $40 \mathrm{~g}$ respectively.

\section{CONCLUSION}

In conclusion, students were successfully introduced to automated flash chromatography, normal phase chromatography and the ELSD detector. They were given the opportunity to experience in an active learning style experiment the influence of column characteristics on chromatographic resolution as well as the different detectors responses on the same sample during the same chromatographic run. This lab experiment allows for a better understanding of i) column loading ii) particle sizes influence iii) mass vs concentration detector sensitivity.

\section{ASSOCIATED CONTENT}

215 Supporting Information

Instructions for students; Additional information for instructors (DOCX)

\section{AUTHOR INFORMATION}

Corresponding Author

*E-mail: danjou@univ-littoral.fr

\section{ACKNOWLEDGMENTS}

Authors would like to thanks the 2018-19 and 2019-20 promotions of the "Analyse Chimique, Contrôle Industriel, Environnement" master for testing the protocol, enriching the reproducibility data and their critical point of view on this work. Authors are also grateful to FCU Côte d'Opale and Université du Littoral Côte d'Opale (ULCO) for financial support. 


\footnotetext{
${ }^{1}$ Still, W. C.; Kahn, M.; Mitra, A. Rapid Chromatographic Technique for Preparative Separations with Moderate Resolution. J. Org. Chem. 1978, 43 (14), 2923-2925.

${ }^{2}$ Jacobson, B. M. An Inexpensive Way to Do Flash Chromatography. J. Chem. Educ. 1988, 65 (5), 459.

${ }^{3}$ Pontén, F.; Ellervik, U. Safe and Efficient Flash Chromatography Equipment for the Research/Teaching Lab. J. Chem. Educ. 2001, 78 (3), 363.

${ }^{4}$ Butler, J. D.; Choung, W.; Kurth, M. J. Flash Chromatography: A Novel Pressurization Apparatus. J. Chem. Educ. 2010, 87 (11), 1265-1265.

${ }^{5}$ Buchi English-language homepage. https://www.buchi.com/ (accessed Jan 31, 2020).

${ }^{6}$ Biotage English-language homepage. https://www.biotage.com (accessed Jan 31, 2020).

${ }^{7}$ Interchim English-language homepage. http://www.interchim.com (accessed Jan 31, 2020).

${ }^{8}$ Naumiec, G. R.; Del Padre, A. N.; Hooper, M. M.; St. Germaine, A.; DeBoef, B. A Modern Apparatus for Performing Flash Chromatography: An Experiment for the Organic Laboratory. J. Chem. Educ., 2013 90 (3), 376-378

${ }^{9}$ Husgafvel- Pursiainen, K.; Kannio, A.; Oksa, P.; Suitiala, T.; Koskinen, H.; Partanen, R.; Hemminki,
}

K.;

Smith, S.; Rosenstock- Leibu, R.; Brandt- Rauf, P. W. Mutations, tissue accumulations, and serum

levels

of p53 in patients with occupational cancers from asbestos and silica exposure. Environ. Mol. Mutagen., 1997, 30, 224-230

${ }^{10}$ Chester, T. L. Recent Developments in High-Performance Liquid Chromatography Stationary Phases Anal. Chem., 2013, 85, 579-589

${ }^{11}$ Swartz, M. HPLC detectors: a brief review. J. Liq. Chromatogr. Relat. Technol., 2010, 33:9-12, 11301150

${ }^{12}$ Miranda, B.; Lawton, N. M.; Tachibana, S. R.; Swartz, N. A.; Hall, W. P. Titration and HPLC Characterization of Kombucha Fermentation: A Laboratory Experiment in Food Analysis. J. Chem. Educ., 2016, 93 (10), 1770-1775

${ }^{13}$ Purcell, S. C.; Pande, P.; Lin, Y.; Rivera, E. J.; Paw U, L.; Smallwood, L. M.; Kerstiens, G. A.; Armstrong, L. B.; Robak, M.; Baranger, A. M.; Douskey, M. C. Extraction and Antibacterial Properties Of Thyme Leaf Extracts: Authentic Practice of Green Chemistry. J. Chem. Educ., 2016, 93 (8), 14221427

${ }^{14}$ Parker, P. D.; Beers, B.; Vergne, M. J. What Is in Your Wallet? Quantitation of Drugs of Abuse on Paper Currency with a Rapid LC-MS/MS Method. J. Chem. Educ., 2017, 94 (10), 1522-1526

${ }^{15}$ Betts, T. A.; Palkendo, J. A. Teaching Undergraduates LC-MS/MS Theory and Operation via Multiple Reaction Monitoring (MRM) Method Development. J. Chem. Educ., 2018, 95 (6), 1035-1039

${ }^{16}$ Urban, P. L. Clarifying Misconceptions about Mass and Concentration Sensitivity. J. Chem. Educ., 2016, 93 (6), 984-987

${ }^{17}$ Mariana, M.; Feiteiro, J.; Verde, I.; Cairrao, E. The effects of phthalates in the cardiovascular and reproductive systems: A review. Environ. Int., 2016, 94, 758-776

18 Waters Corporation. 2424 Evaporative Light Scattering Detector Operator's Guide. https://www.waters.com/webassets/cms/support/docs/71500121802rb.pdf (accessed Jan 31, 2020). 